\title{
¿CIUDADANÍA O AYUDA SOCIAL? del diseño de la política pública a la experiencia de las mujeres beneficiarias asistenciales en Chile
}

\author{
Marco Ceballos*
}

\begin{abstract}
En torno al caso chileno, este artículo se propone interrogar el sentido que adquiere la noción de ciudadanía en el marco de las actuales políticas de asistencia social en Chile. En un primer momento, se analiza la evolución reciente de éstas así como las lógicas y procesos que explican la emergencia y transformación del conocido dispositivo "Chile Solidario" (CHS), poniendo énfasis en la constelación de ayudas sociales que se consolida a su alero. En un segundo momento, se discute el concepto de ciudadanía desde una perspectiva sociológica, no formal ni normativa, y se intenta mostrar, a partir de testimonios de mujeres beneficiarias de políticas de asistencia social en Chile, el contenido empírico de una ciudadanía asistencial como prevalece en sus experiencias de vida.

Palabras-clave: Política social. Asistencia social. América Latina. Chile Solidario. Conditional Cash Transfer Programs (CCTP).
\end{abstract}

\section{CHILE SOLIDARIO Y LA ASISTEN- CIA SOCIAL EN CHILE}

La experiencia chilena de transformación de lo social, desde los años 1980 en adelante, es emblemática para América Latina. Chile fue pionero en la exploración, la implementación y la racionalización de los procesos de redefinición de lo social y del rol del Estado que se consolidaron en la región desde inicios de las reformas y ajustes estructurales y de la crisis de la deuda externa. Este artículo no se propone trazar la historia de esas transformaciones; al respecto, la literatura es vasta. Como se desarrolla más adelante, el panorama actual de la política social en América Latina resulta de una serie de procesos de transformación burocrática, económica y social, en un marco de desarrollo subsidiario del Estado de expansión del mercado (Mesa-Lago, 2000).

Identificamos, al menos, tres herencias principales que explican la complejidad de lo

* Doutor em Sociologia. Professor da Escola de Sociologia, Faculdade de Humanidades e Ciências Sociais da Universidad Andrés Bello, UNAB, Viña del Mar, Valparaíso. Quillota 980, $5^{\circ}$ piso. Viña del Mar - Valparaíso. marco.ceballos@unab.cl social y sus instituciones en América Latina. En primer lugar, entre las décadas de 1920 y 1970, se desarrollaron regímenes de protección y de seguridad social, en un contexto de "Estados de compromiso", cuya pretensión era instituir derechos sociales en la línea de los modelos europeos; en los hechos, este proceso contribuyó de manera importante a fragmentar los estatus sociales (Barba, 2003).

En segundo lugar, desde el desmantelamiento parcial del Estado de compromiso latinoamericano, entre fines de los años 1970 y mediados de los años 1990, según los países, la política social tendió hacia la híper-focalización (Lautier, 2003) del gasto social público en torno a la cuestión de la pobreza y, más concretamente, respecto de ciertas categorías de pobreza y determinadas situaciones consideradas prioritarias. Al mismo tiempo, se crearon condiciones favorables para el desarrollo de mercados de prestaciones sociales, configurándose una tendencia a la dualización en el acceso y goce de derechos sociales (Duhau, 1997).

En tercer lugar, desde finales de la década de 1990, marcada por los efectos de la crisis asiática y por la adhesión a los "objeti- 
vos del milenio" de la ONU para la reducción de la pobreza, las políticas sociales han buscado mejorar su performatividad mediante un aumento controlado del gasto social público, susceptible de generar impacto sobre las economías familiares de los pobres, multiplicándose la oferta en materia de ayudas sociales y persiguiendo la institucionalización de sistemas integrados de administración de lo social (como los catastros únicos de beneficiarios y el control de "fraudes") en el contexto de una importante sectorización de la política pública.

\section{El carácter fragmentario de la políti- ca asistencial en Chile}

Desde la crisis asiática de 1998, que golpeó duramente a la economía chilena, se vienen desarrollando esfuerzos de racionalización de la política social cuyo objetivo no ha consistido únicamente en generar impactos positivos en los hogares pobres, sino también coordinar, de mejor modo, la oferta social "pro-pobres", fuertemente fragmentada en términos institucionales, referenciales y financieros. En esta línea, durante la administración del Presidente Ricardo Lagos (2000-2006), se creó el "Sistema de protección social Chile-Solidario" (en lo เ2 sucesivo, CHS) dependiente del Ministerio de 亏ิ Planificación (MIDEPLAN), hoy Ministerio de Desarrollo Social (MDS). CHS absorbió, al meสี่ nos nominalmente, varios subsidios sociales directos e indirectos creados bajo la dictadura de Pinochet, como las Pensiones Asistenciales (PASIS) - hoy llamadas Pensión Básica Solidaria (PBS) - y el Subsidio Único Familiar (SUF), a ambos de responsabilidad del Ministerio del i Trabajo y Previsión Social, así como el Sub-

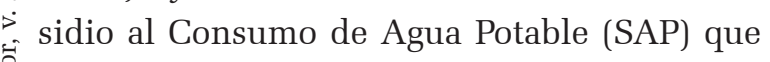
otorga el Ministerio del Interior.

CHS representa, en realidad, un híbrido institucional, entre un programa social y un sistema de programas sociales: al mismo tiempo que asume tareas de coordinación de la oferta social pública de tipo asistencial, crea un dispositivo propio de transferencias monetarias condicionadas, ${ }^{1}$ cuyo horizonte, jamás alcanzado, consiste en ayudar a sostener económicamente, durante una cantidad limitada de tiempo, cerca de 250 mil familias clasificadas en situación de extrema pobreza (Cf. Lautier, 2011). CHS consigue sólo parcialmente unificar las operaciones de algunos de los numerosos programas sociales focalizados en los pobres. Concretamente, no consigue hacer que éstos compartan los mismos criterios de asignación de recursos, las mismas definiciones de público-objetivo, las mismas condicionalidades y procedimientos de control de las "contrapartidas" y las "corresponsabilidades", las mismas duraciones de tiempo de participación, los mismos léxicos.

CHS no resuelve realmente el problema de la fragmentación de públicos y de la dispersión de las ayudas sociales. De hecho, este programa opera simultáneamente a nivel nacional, local y sectorial. A nivel nacional, MIDEPLAN y Fondo Solidario de Inversión Social (FOSIS), organismo semi-dependiente de este ministerio, tienen la tarea de filtrar a los beneficiarios, de gestionar algunas transferencias monetarias y de coordinar un programa de "acompañamiento familiar" llamado "Puente", que consiste en visitas domiciliarias llevadas a cabo por trabajadores sociales que despliegan, al menos teóricamente, dinámicas de intervención "psicosocial" hacia los miembros de hogares pobres.

Si bien la gestión técnica del programa "Puente" es responsabilidad de FOSIS en el nivel central, en principio, su ejecución es responsabilidad de los municipios en el nivel local. Según los casos, hay municipios que no consiguen desvincularse de la gestión directa de FOSIS, por razones de escasez de funcionarios, de recursos o de capacidad operativa. Los municipios, además, tienen la tarea de producir la información social de sus territorios con que se alimenta el catastro nacional ${ }^{1}$ CCTP, por su sigla en inglés: Conditional Cash Transfer Programs. 
de beneficiarios sociales de FOSIS y del MDS. Con considerable dificultad, los municipios intentan articular sus propias ayudas sociales y sus propias estrategias contra la pobreza, variables en función de sus recursos en un marco de descentralización de larga data (fines de los años 1970), con el programa "Puente" y con CHS. Los resultados son muy heterogéneos según los territorios, pero también según las capacidades administrativas de las burocracias locales y según el signo político de cada municipio. También encontramos entrelazadas una dimensión sectorial y otra de gestión local a nivel de las intervenciones en materia de educación escolar pública y de salud primaria, que son de responsabilidad de los municipios antes que del gobierno nacional. ${ }^{2}$

A nivel sectorial, aparte de la gestión de los subsidios específicos mencionados más arriba (SAP, SUF y PBS), CHS ha intentado implementar un sistema de acceso preferente para sus beneficiarios a servicios sectoriales, como los subsidios a la vivienda, la atención primaria de salud y los programas de empleo y formación laboral del Ministerio de Economía. Su débil logro se explica porque dichas políticas específicas no comparten las mismas definiciones de público prioritario de CHS ni responden a las mismas estrategias de cobertura. Tres ejemplos: la expansión efectiva de la atención de salud primaria con foco en la salud materno-infantil, en los últimos diez años, en Chile, responde a una política de mediana data (2003) de Garantías Explícitas en Salud (AUGE-GES) de carácter universalista, aunque dentro de un marco de dualización de la salud; ${ }^{3}$ la política de subsidios a la vivienda

${ }^{2}$ Desde 1981, en Chile, la gestión de los establecimientos públicos de educación escolar y de los establecimientos de salud primaria corresponde a los municipios. Actualmente, uno de los temas principales de la agenda pública se refiere, justamente, a la trasferencia de estos servicios bajo la responsabilidad directa de los ministerios correspondientes.

${ }^{3}$ Por una parte, la población pobre y de ingresos medios -bajos accede a los seguros públicos de salud en Fondo Nacional de Salud, FONASA, y se atiende en la red de establecimientos públicos (en crisis financiera, profesional y de infraestructura desde los años 1980). Por otra parte, la población de ingresos medios y altos cotiza en los segu- sigue criterios propios de calificación de beneficiarios, considerando distintas modalidades para públicos específicos según capacidades de endeudamiento, $y$ ha adecuado fuertemente sus programas a eventos catastróficos de los últimos años (terremoto en 2010, terremoto en 2014, mega-incendio en Valparaíso en 2014); los programas de formación y colocación laboral son de alcance limitado, dependen de la demanda del mercado laboral y de las características económicas de los territorios.

Así, entonces, el acceso prioritario de beneficiarios de CHS a políticas sectoriales es de difícil realización dentro de un esquema híbrido de fragmentación de públicos, de dualización de servicios y de sectorización de políticas y concepciones de lo social. Esa arquitectura refleja, justamente, que el acople entre los dispositivos de asistencia a la pobreza extrema, con todo el desarrollo en términos de redes de protección social para pobres que ello ha significado, y las políticas propiamente de seguridad social (salud, educación, vivienda y seguridad social) no ha tenido lugar.

De este modo, poco después de su implementación en 2002, CHS es objeto de un diagnóstico bastante crítico respecto de su falta de impacto y del fracaso en sus esfuerzos de coordinación de la oferta social-asistencial a distintos niveles administrativos y territorios. También es objeto de críticas y denuncias de clientelismo poco fundadas por parte de sectores políticos de derecha. La Presidenta Michelle Bachelet, durante su primer gobierno (2006-2010), toma distancia respecto de CHS, aunque, en los hechos, lo transforma y lo refuerza. Su gobierno crea el "Sistema Intersectorial de Protección Social" o "Red de Protección Social”, con la intención de subsanar una serie de vacíos operacionales de CHS en cuanto a intervenir sobre determinadas trayectorias sociales de riesgo o vulnerabilidad: la maternidad y la pequeña infancia, la vejez, las "situ-

Previsional, ISAPRES, propiedad de capitales nacionales e internacionales, y se atienden en el sistema privado de clínicas y centros médicos de mediana y alta gama. 
aciones de calle" o la separación familiar por casos de encarcelamiento. Se busca, entonces, abordar estas problemáticas por medio de intervenciones en materia de salud, de escolaridad y educación pre-escolar, de asociatividad local, de obras públicas, etc.

Los nuevos programas que vienen a complementar la acción de CHS recurren, ellos también, a su sistema de clasificación de beneficiarios. Hay que insistir en esta idea: en última instancia, el dispositivo que asegura una cierta coherencia en la atribución de las ayudas sociales en Chile radica en el sistema de clasificación de las vulnerabilidades sociales desarrollado y gestionado por CHS. Se trata de un modelo de tratamiento de la información social que proveen los municipios a través de un instrumento llamado "Ficha de Protección Social", ${ }^{4}$ que "mide la situación socioeconómica y la vulnerabilidad de las familias chilenas de manera más rigurosa, garantizando un acceso más equitativo a los beneficios sociales otorgados por el Estado". ${ }^{5}$ Allí se consolida la información individual y por grupos familiares, respecto de sus situaciones económica, demográfica, relacional, sanitaria, escolar, territorial, familiar, laboral, habitacional, etc. Esta medición multivariada, compleja, de la vulnerabilidad social produce un output de เ2 puntajes familiares de vulnerabilidad, o de స్ "habilitación social". Su metodología de cálculo ha sido modificada reiteradamente en la สี última década y media. Este ranking de pobre¿ za asegura, o niega - según cada caso -, a las familias pobres, un estatus de validez ante los programas sociales. Se trata de un sistema de c clasificación que califica o descalifica a las faa milias en función de sus necesidades y riesgos $\stackrel{\infty}{\sim}$ sociales (Paugam, 2012). Sirve también para

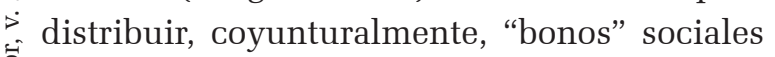
(transferencias monetarias directas no condicionadas), por ejemplo, con motivo del alza de

Se crea a principios de los años 1980, con el nombre "Ficha de Caracterización Social".

5 Ver http://www.nuevaficha.gob.cl/ficha.php. Vista el $30 / 01 / 14$ los precios de los alimentos y del petróleo en 2007 y 2008, luego de la crisis financiera internacional de 2008 y 2009, con ocasión del terremoto de 2010 y del gran incendio en Valparaíso en 2014 o, incluso, para hacer frente a los gastos escolares de las familias a inicios de cada año, como ocurre casi ininterrumpidamente desde 2009.

\section{Una constelación de ayudas socia- les}

CHS y, más ampliamente, la política social-asistencial en Chile carecen de indicadores de impacto. No podemos afirmar, a ciencia cierta, los efectos que tienen sobre la pobreza o la extrema pobreza. En un contexto de tecnocratización creciente de la gestión pública, esto plantea un problema de credibilidad. Comparativamente, estudios de impacto asociado a este tipo de programas, ${ }^{6}$ en América Latina, tienden más bien a confirmar la insignificancia estructural de sus efectos tanto sobre las tasas de pobreza y extrema pobreza como sobre la desigualdad de ingresos. La mejoría global de indicadores sociales que conocen varios países de la región entre mediados de la década de 1990 y mediados de la década de 2000 habría respondido principalmente a factores macro-económicos, productivos, demográficos o redistributivos, como, por ejemplo, el aumento real de los salarios mínimos legales (Barros, Henríquez, Mendoça, 2001; Melo-Borges, Sarmento-Seixas, 2006; Cf. Soares et al., 2007; Marques-Pereira, 2004).

Con el triunfo presidencial de Sebastián Piñera (2010-2014), apoyado por una coalición de derecha neoconservadora, CHS se articula con un dispositivo de transferencias monetarias condicionadas llamado "Ingreso Ético Familiar” (IEF). El IEF asigna recursos complementarios a otros programas de transferencias monetarias en función de ciertos "méritos" específicos que las mujeres, beneficiarias so-

${ }^{6}$ Para una crítica a la tipología de los CCTP, ver: Ceballos; Lautier, 2013. 
ciales, son capaces de exhibir: asistencia de los hijos a la escuela, rendimiento escolar de los hijos, asistencia de los hijos a los controles de salud, generación autónoma de ingresos ("Bono al Trabajo de la Mujer").

Entonces, se transforman algunos criterios de asignación de las ayudas sociales, limitando la posibilidad de acumular determinados beneficios así como poniendo el acento - al menos en los relatos políticos - en el mérito individual y en la empleabilidad femenina, por sobre el reconocimiento de la vulnerabilidad y la práctica de la solidaridad. Con ello, el panorama de las ayudas sociales (sus montos, sus condiciones de acceso, sus duraciones, la vinculación entre programas, sus funcionamientos y burocracias, inclusive sus referenciales) se hace aún más complejo. No obstante, CHS sigue siendo "la puerta de entrada" a una oferta social pública destinada a ayudar a sostener las economías familiares de los pobres. Y esta oferta se entrelaza en ocasiones, no se toca en otras, con las ofertas sectorial y local en materia de salud, de educación, de vivienda y de asistencia.

El campo de la asistencia social en Chile constituye, de este modo, parafraseando a Robert Castel, una "constelación [...] extraordinariamente diversificada de prácticas” (Castel, 1995, p. 47): una constelación de ayudas sociales. Sus instituciones, sus normas, sus objetivos, sus procedimientos, sus justificaciones y argumentaciones, sus prácticas y modelos de intervención, sus recursos y el origen de éstos constituyen un territorio extremadamente confuso y amorfo, cuyas fronteras resultan irrepresentables.

Este fenómeno es característico de un Estado subsidiario - en su sentido doctrinario liberal - en tanto persigue "limitar al máximo todo tipo de intervención pública en la economía” (Loo-Gutiérrez, 2009, p. 404). En América Latina (por ejemplo, en la Constitución Política de Chile, 1980), se redujo la intervención productiva y redistributiva del Estado en favor de la "iniciativa individual y privada" y de la "libertad de elección" como condiciones necesarias para el desarrollo de la mercantili- zación de lo social. Esto es válido, también, en materia de salud, de educación escolar, técnico-superior y universitaria, en materia de desregulación del empleo y el trabajo, en materia de ahorro para la jubilación, y en numerosos otros aspectos.

De lo anterior resulta un panorama social -asistencial cuyo diseño, en Chile, no puede ser comparado, realmente, con un eventual "derecho a la asistencia social" en los términos fijados, por ejemplo, por la Constitución Política del Brasil de 1988, ni por sus Leyes Orgánicas de Asistencia Social (LOAS) de 1993, al menos a nivel de su diseño político. Por el contrario, la historia de la asistencia social en Chile muestra cuán enraizadas están las viejas distinciones entre "verdaderos" y "falsos" pobres (Illanes, 2006), muy lejano de una "reconceptualización" del trabajo social como aquella ocurrida en Brasil en los años 1970. La fórmula "asistencia social”, de uso corriente durante el siglo XX, prácticamente desapareció del léxico latinoamericano desde los años 1990 en adelante, a excepción de Brasil, donde se crearon instituciones de asistencia con el objeto de garantizar derechos progresivos, incondicionales, permanentes y categoriales, al menos en la letra. Lo distintivo del caso brasilero, en este sentido, es haber definido, desde el espacio político y social, el objeto "asistencia social" como un campo de intervención social y de reconocimiento de derechos. En el resto de América Latina, esta fórmula fue tempranamente abandonada por el campo técnico-político de lo social, cargada por el estigma del "asistencialismo" de los años 1980, instalando, en su lugar, un relato de la asistencia social en términos de "protección social" y de "redes de seguridad".

Con la emergencia de los CCTP y de una nueva generación de programas sociales, ciertamente se amplían los estrechos márgenes de acción de las políticas híper-focalizadas de los años 1980 y 1990, pero no llegan a implementarse sistemas de protección social propiamente tal, puesto que la focalización de los programas sigue siendo un fenómeno real 
a pesar de su considerable masificación en algunos países. Se trata, entonces, del desarrollo de una oferta diversificada y compleja, pero, sobre todo, selectiva, que busca incorporar a las familias pobres en determinadas redes de prestaciones sociales masivas, pero fragmentarias. Lo que se configura, en la práctica, es un territorio insondable de acción pública ocupado por gran número de dispositivos de ayuda social ("constelaciones") que intervienen ex -post, fragmentariamente, discontinuamente y en función de escenarios críticos o estratégicos, priorizando a mujeres, a niños y a ancianos en tanto poblaciones con mayor acumulación de riesgos sociales. Se trata de ayudas específicas (monetarias, subsidios indirectos $\mathrm{y}$, en ocasiones, especies como materiales de construcción, canastas de alimentos, colchones y camas, etc.), destinadas a poblaciones de riesgo clasificadas en función del territorio, de sus configuraciones familiares, de sus ingresos y su participación en el trabajo, del género, la edad, las cargas sociales y toda una métrica compleja de la vulnerabilidad social.

\section{Una asistencia social de "género"}

La asistencia contemporánea en Chile, ـ si pretendemos analizar el fenómeno en un จे plano estructural, está configurada de modo de 荌 abstenerse de actuar de manera voluntarista sobre la cuestión redistributiva en el contexto de una de las economías más desreguladas del mundo y de uno de los países más desiguales $\because$ de América Latina. A través de lo social-asis$\stackrel{2}{\infty}$ tencial, el Estado transfiere, progresivamente, $\therefore$ la responsabilidad de los riesgos sociales hacia ㅇ los propios individuos y, más concretamente, $\overrightarrow{~ h a c i a ~ l a s ~ m u j e r e s ~ p o b r e s . ~ L o ~ a n t e r i o r ~ s e ~ s o s-~}$ tiene, además, en una gestión biopolítica de la pobreza (Rojas, 2012), a través de programas de "acompañamiento familiar" como "Puente" que implementan modelos de intervención psicosocial que se han generalizado en distintos países de la región.
La cuestión sobre la ciudadanía "social”, entonces, ya no hace referencia a un sujeto masculino típico que, a través del trabajo formal, tiene acceso, junto a su familia, a un estatus garantizado de protección y reconocimiento sostenido por el Estado y las instituciones mutualistas de las que es contribuyente. En este contexto de desmantelamiento de larga data de las instituciones y estructuras de seguridad social en América Latina, de precarización del estatus del empleo y de transformación en la configuración de las familias junto al desarrollo de una nueva generación maleable o modular de programas sociales (Sojo, 2003), emerge un nuevo estatus social: el de las mujeres jefas de familia en situación de pobreza económica y de precariedad en el acceso a la educación, a la salud, al trabajo, a la vivienda, principales beneficiarias de las ayudas sociales. Estas políticas sociales “pro-pobres” producen una rehabilitación discursiva y argumental de la ciudadanía en torno de la figura de las mujeres. Ello se transforma en un referencial narrativo de primer orden de la política social en todos los países de América Latina, como ejemplifican los relatos de cuatro ex-presidentes en torno a cuatro conocidos programas de transferencias monetarias condicionadas:

En muchas situaciones, la madres, sin la ayuda de nadie, cargan el peso de toda la familia. [...] Hoy, aquí, estamos dando un paso extraordinario en nuestro camino. 'Bolsa Familia' es una gran evolución de los programas sociales en Brasil. [...] Su propio nombre deja en claro el objetivo del programa: dar protección integral a toda la familia y no sólo a algunos de sus miembros (Presidente Luíz Inácio Lula da Silva, discurso de promulgación de la ley que crea el programa “Bolsa Familia”, octubre de 2003).

'Oportunidades' nació para apoyar a las madres, a las madres que cuidan con cariño a sus hijos, que cuidan el gasto de la familia, que llevan a los hijos al doctor y se preocupan para que vayan a la escuela" [...]. El programa 'Oportunidades' se convierte en un instrumento permanente, en un instrumento de dignificación, en un instrumento de desarrollo para nuestros hijos (Presidente Vicente Fox, Discurso en un acto de entrega de beneficios del programa 'Oportunidades', San Luís de Potosí, septiembre de 2005). 
[Nuestro objetivo consiste en]... proteger a las familias en situación de vulnerabilidad. [...] El 'Plan Familias' es considerado central en nuestra política de Estado, porque busca promover los valores que cohesionan, articulan y hacen posible una vida armoniosa en familia. Contempla acciones de protección, prevención, asistencia, acompañamiento y promoción. El eje es la mujer como movilizadora y transformadora. (Presidente Néstor Kirchner, Discurso ante la Asamblea General, marzo de 2005).

Son muchas las tareas que se han hecho en Chile Solidario. Estuve en Curacaví, con un grupo muy significativo de personas que participan en esa comuna, y allí habló doña Carmen Vera, viuda, con dos hijos, es temporera. Ingresó a Chile Solidario en agosto de 2002. Hoy, doña Carmen está cursando de primero a cuarto año básico. Me explicó, en una nota que me envió, que antes no sabía leer ni escribir, y las palabras que me dijo fueron: 'hoy escribí por primera vez. Coloqué en un papel mis ideas y las cosas que quería contarle'. Yo quiero aplaudir a doña Carmen, y a todos los miles que como ella se atreven a decir "sí, no sé leer y escribir.” [...] Estuvimos en Valdivia hace un par de meses atrás y allí habló Sandra Aguilar, casada, siete hijos. Habló diciendo que vivía de allegada en una mediagua donde no había agua potable. Nos emocionó porque dio las gracias, nos emocionó porque pensó que tenía una posibilidad de surgir a partir de una sociedad que le tendió la mano en Chile Solidario (Presidente Ricardo Lagos, Mensaje Presidencial ante el Congreso Nacional, mayo de 2003 y mayo de 2004).

El imaginario político que alimenta los relatos sobre lo social en torno a las nuevas políticas de asistencia en la región moviliza enunciados de "género" que quedan en evidencia en los extractos anteriores, por ejemplo en torno a la glorificación del sacrificio cotidiano de las madres y las mujeres, y a la valorización de la familia como espacio de gestión femenino y como unidad social básica, elementos que encontramos en todos los niveles de desarrollo e implementación de estas políticas. Las políticas de asistencia empalman su relato social y su estrategia de intervención con la construcción tradicional de género predominante en nuestras sociedades latinoamericanas, en torno a la responsabilización doméstica y familiar de las mujeres, y en torno a la pobreza como lugar por excelencia donde ese patrón de género tiene lugar. ¿Qué clase de ciudadanía social puede pretenderse cuando las políticas de asistencia social y, más ampliamente, el acceso de la población pobre a la política social, ${ }^{7}$ utilizan y reproducen las construcciones tradicionales y desigualitarias de género predominantes en la sociedad, institucionalizándolas? Se trata de una forma de sujeción o de subordinación de género como instrumento de desarrollo familiar y del bienestar social.

\section{Ciudadanía y ayudas sociales}

Las nuevas políticas sociales latinoamericanas movilizan un discurso de reconocimiento de derechos sociales que adquiere un carácter más moral que jurídico y, en consecuencia, más teórico que realizable (Garretón, 2010). En la actualidad, encontramos una concepción desmaterializada de la ciudadanía, que se aleja fuertemente de lo que había sido su construcción histórica en Europa. "La invención de lo social”, en Europa, ha sido explicado así: "lo social surge como una invención necesaria para hacer gobernable una sociedad que opta por un régimen democrático [... dado] el contraste entre la proclamación de una soberanía igualitaria y el sometimiento económico de las clases más numerosas. [...] ¿Cómo reducir esta brecha entre el nuevo fundamento del orden político y la realidad del orden social, con la finalidad de asegurar la credibilidad del primero y la estabilidad del segundo?" (Donzelot, 1984, p.13, p. 32-33). Lo social fue la respuesta europea dada a esa pregunta por las contradicciones internas de los sistemas sociales que emergen con la modernidad occidental.

La ciudadanía hace alusión, en primer término, a un estatus social de pertenencia y participación en un estado de derecho. Cono-

7 Mayoritariamente, las mujeres también asumen otras cargas de titularidad y responsabilidad familiar frente al Estado, más o menos explícitas según los casos, por ejemplo respecto de los programas de acceso a los subsidios a la vivienda, respecto de la escolarización de los hijos y las políticas de becas, respecto del acceso al sistema de salud de niños y ancianos, etc. 
cemos ampliamente la distinción socio-histórica propuesta por T. H. Marshall, hace más de medio siglo, entre ciudadanía civil, política y social (Marshall, 1997). El autor relaciona, a través de su teoría, las fases del desarrollo europeo moderno con la extensión de derechos que transforman el vínculo entre los individuos, la sociedad y el Estado. A lo largo de los siglos XIX y XX, Europa habría vivido el paso de una concepción de ciudadanía restringida a una de ciudadanía amplia, de una ciudadanía formal a una ciudadanía activa, a través de instituciones efectivas de solidaridad social. Los ciudadanos europeos, de acuerdo a esta periodización ideal, gozarían de un estatus de pertenencia social históricamente construida y que constituye una dimensión dinámica de su vida social, una experiencia empírica, antes que una pura cuestión enunciativa o formal.

Más allá de los grandes esfuerzos intelectuales en este sentido, la transferencia de categorías analíticas desarrolladas en Europa hacia América Latina es siempre objeto de problema. Numerosos autores constatan, en América Latina, las enormes brechas sociales que producen las desigualdades de género, socioeconómicas, étnicas o territoriales, independientemente de los sistemas legales reconocedores de derechos universales. Lo propio de una ciudadanía efectiva, en América Latina, 亏े. sería, entonces, su inefectividad. Hablamos, entonces, de un “déficit de ciudadanía”, entenสี dido como un estado de "precariedad [...] en el acceso a los recursos básicos para el ejercicio de los derechos y los deberes" (Moreno, 2003, p. 1; Moreno, 2000). Se subrayan, entonces, "las dificultades que implica la construcción a de ciudadanía en sociedades marcadas por la $\stackrel{\infty}{\sim}$ heterogeneidad estructural, por las grandes $\vec{b}$ brechas sociales y por los conflictos culturales recurrentes" (Fernández, 1998, p. 13). Dada la "situación de falta de ciudadanía a raíz de las condiciones de pobreza y grandes desigualdades, así como de la existencia de numerosos grupos cuya ciudadanía no es defendida ni definida" (Castro, 2009, p. 8), algunos autores proponen referirse a una "ciudadanía de baja intensidad” (O’Donnell, 1997) o "ciudadanía regulada” (Dos Santos, 1979).

Esta realidad es más compleja a la luz del desarrollo explosivo de los mercados. De acuerdo a una tesis muy difundida en los años 1990, en América Latina se produce una profunda mutación de lo que entendemos por ciudadanía, a raíz de la emergencia del mercado como lugar de participación y de pertenencia social: "muchas de las preguntas propias de los ciudadanos - adónde pertenezco, cuáles son mis derechos, como me informo, quién representa mis intereses - encuentran respuesta en el espacio del consumo privado de bienes y medios, antes que a través de reglas abstractas de la democracia o de la participación colectiva en los espacios públicos" (García-Canclini, 1995, p. 13). La noción de ciudadanía se ha transformado, de esta forma, en un objeto problemático en América Latina, donde se entremezclan una noción política, que remite al Estado y a lo público, y una dimensión económica, que remite a la participación de las personas en el mercado. La ciudadanía social ya no es sinónimo de desmercantilización de las relaciones sociales, sino que reflejo de una gran heterogeneidad de estatus sociales y modos de participación social.

Si la idea de ciudadanía igualitaria surgió con motivo de un proceso social y político de regulación del liberalismo económico, la desregulación económica debiera, entonces, ser igualmente analizada en términos de una fragmentación o diferenciación de la ciudadanía (Cf. Fariñas, 1999; Young, 1996), de hibridez estructural de lo social (Gough, 2004) y de multiplicidad de estatus sociales (Lautier, 1993). Y, dentro de ello, es pertinente y necesario observar el estatus que le reconoce el Estado (y la sociedad) a las mujeres pobres, extremadamente pobres y (o) vulnerables, como sujetos típicos de las ayudas asistenciales. En los últimos años, se ha perfilado, con claridad, un estatus específico de pertenencia social de las mujeres asistidas socialmente por el Esta- 
do: ellas son beneficiarias y titulares de ayuda, a título de bisagras funcionales de las políticas de intervención social familiar. Su estatus de ciudadanas, entendido como una condición de protección y reconocimiento sociales (Paugam, 2012), representa un territorio problemático, como veremos más abajo.

\section{La clasificación de la vulnerabilidad}

El Sistema Integrado de Información Social en Chile (SIIS), operado por el MDS FOSIS, se alimenta gracias al levantamiento municipal de información vía "Ficha de Protección Social". La aplicación de esta "ficha", programada desde el nivel central, ha ocurrido de manera discontinua y, en el último tiempo, han transcurrido largos períodos de cese, superiores al año en algunos casos (2012-2014). Para efectos de calificarse en CHS y en otros programas sociales y "bonos" de emergencia, los demandantes y beneficiarios deben haber sido encuestados recientemente $\mathrm{y}$, a los pocos meses, tener asignado un puntaje de vulnerabilidad por unidad familiar. A mayor vulnerabilidad, menor puntaje y mayor probabilidad de ser seleccionados. Ser mujer y madre de menor(es) de edad, con adultos mayores o personas con discapacidades o enfermedad crónica a cargo, sin pareja hombre en el domicilio, sin ingresos formales, no propietaria de la vivienda ni del sitio, con baja cantidad de años de estudio, son algunos de los principales factores de vulnerabilidad que determinan un bajo puntaje.

En sus treinta y cinco años de existencia, ${ }^{8}$ este instrumento y su tratamiento estadístico han alcanzado un alto grado de desarrollo. En la actualidad, sus datos se combinan con aquellos de la encuesta de hogares de Caracterización Socio-Económica Nacional (CASEN), de responsabilidad directa del MDS y cuya

${ }^{8}$ En 1979, bajo la dictadura cívico-militar encabezada por Augusto Pinochet, se crea el primer instrumento de levantamiento municipal de información social, la ficha de "Caracterización Social" (CAS). En democracia, "la ficha" ha experimentado numerosos cambios metodológicos. aplicación - externalizada en la Universidad de Chile hasta 2006 - ha sido transferida al Instituto Nacional de Estadísticas (INE).

Los funcionarios técnicos y estadísticos del MDS y de CHS entienden producir una construcción estadística de la pobreza, funcional a las necesidades de las políticas públicas y del gasto social programado․ Las recetas públicas disponibles operan como un factor determinante de la capacidad de cobertura de estas políticas. La atribución efectiva de las ayudas sociales depende, entonces: a) del puntaje del núcleo familiar resultante de una medición multidimensional de la pobreza y de su ubicación en el ranking acorde a criterios de vulnerabilidad predefinidos; b) de la priorización del territorio y la partida fiscal que se le asigne en función de ello y, yendo más lejos en el análisis, de definiciones políticas de gasto social e inversión territorial de la Ley de Presupuesto en el Parlamento; c) y de la capacidad discrecional y profesional de la administración territorial-asistencial (Lipsky, 1980) para orientar parte de las ayudas a determinados usuarios de la asistencia social - lo que analizamos más adelante - así como del manejo de su información social que realizan los demandantes de ayudas.

La elevada complejidad que detenta, actualmente, la metodología de identificación, caracterización y ranking de hogares vulnerables se justifica, en parte, desde la perspectiva de los equipos técnicos responsables, como una garantía de objetividad del proceso a la vez que como un recurso para reducir los "fraudes": las personas no consiguen, en principio, delinear una estrategia de respuestas que les ayude a obtener puntajes más beneficiosos, en la medida en que desconocen el peso de los factores sobre la medición de la vulnerabilidad social. Mediante un artefacto estadístico y metodológico que opera como herramienta de poder, el sistema de cálculo de puntajes de vulnerabilidad social intenta garantizar una certeza cuan${ }^{9}$ Entrevista con equipo técnico-estadístico del MIDEPLAN (actual MDS), Santiago, 2008. 
titativa en la medición individual y familiar de los riesgos sociales, al mismo tiempo que mantiene parte de su saber bajo llave.

\section{La selección como experiencia de exclusión}

Las mujeres beneficiarias o demandantes de la asistencia social, con mayor o menor conocimiento, intuición o ayuda, intentan regular la información social que proveen, por ejemplo negando la existencia de un hombre adulto y proveedor en el hogar, no declarando ingresos autónomos informales o presentando condiciones de vulnerabilidad que les parecen valederas, pero que no tienen necesariamente peso sobre sus puntajes (por ejemplo, la condición de pareja joven desempleada, o con niños pequeños). Pero la calificación a través de este indicador multidimensional de la pobreza no empalma, necesariamente, con la definición experiencial de las beneficiarias de las ayudas. Para ellas, el cálculo de la vulnerabilidad social utilizado por las políticas sociales chilenas está desviado, en un sentido estadístico y político.

En las entrevistas y grupos de discusión realizados con mujeres beneficiarias de pro늘 gramas social-asistenciales en Chile, ${ }^{10}$ emerge 亏े un tópico, una unidad significativa del lenguaje (Ibáñez, 1994), que alcanza la mayor satuฐี ración narrativa. Se trata del problema de la ¿ selección de los beneficiarios, "el problema del puntaje”. El sistema de puntaje de vulnerabili$\rightarrow$ dad, que condiciona el acceso a cualquier prog grama de asistencia social, desde las canastas ¿ básicas de alimentos que distribuyen algunos $\stackrel{\text { i }}{\infty}$ municipios hasta la postulación al subsidio a

$\ddot{0}{ }^{10}$ Serie de entrevistas y grupos de discusión con mujeres beneficiarias de CHS y con funcionarios del programa $\stackrel{\pi}{\perp}$ 'Puente' a nivel municipal en siete comunas de la Región ๙ Metropolitana de Santiago (Peñalolén, La Florida, Puente Alto, Nuñoa, Santiago Centro, Lo Prado) y Lampa, entre T2 2010 y 2013, en el marco del proyecto ANR-10-SUDS-0008

U "Latinassist": participaron Carolina Rojas (co-investigado-

8 ra) y los asistentes de investigación y tesistas de la Universidad Academia de Humanismo Cristiano Francisca Miralles, Francisca Riquelme, Héctor Salazar, Daniel Aravena, Manuel Cortés, Leandro Sanhueza y Nicole Barriga. la vivienda, ${ }^{11}$ representa un problema de justicia social.

El puntaje es el problema. [...] Mi hijo menor no tiene derecho a almorzar en la escuela porque su puntaje es demasiado alto (Elizabeth).

Obtuve once [mil puntos], demasiado para que mi hija pueda acceder al programa "Trabajo de jóvenes" (Agustina).

[...] la gente como nosotros se supone que tenemos un puntaje alto, que no somos tan pobres como lo somos en realidad y que no tenemos tantas necesidades como las que tenemos en verdad. Estamos entre la espada y la pared: no tenemos subsidio [para la vivienda] ni tampoco crédito bancario (Denisse).

En verdad, yo no sé qué miden en sus encuestas. Mi pareja y yo vivimos donde mi suegra. No tenemos nada de nosotros, nada, absolutamente nada. Nos hicieron un montón de preguntas. La señora tomaba y tomaba notas. Ahora acabo de ir a consultar mi ficha iy nos dieron más de diez mil puntos! ¿Pero cómo? No nos alcanza para comer, no llegamos a fin de mes, ¿y esos puntos de dónde salen? (Tamara)

No saben medir, su ficha no sirve para medir todo lo que tienen que medir, no hacen ningún esfuerzo por darse cuenta de nuestra realidad (Tania).

Yo jamás pensé que me iban a elegir para el 'Puente'. Jamás, jamás pensé una cosa así. Tuve un puntaje súper bajo (Ruth).

No todo el mundo está invitado al 'Puente', no todos tienen esa suerte. Yo, es porque mi puntaje es muy bajo (Isabel).

Un día, alguien te visita, te toca la puerta y te dice "te lo dieron”. Es como la lotería. (Paty).

Ganarse el "Puente” es un lujo (Paty).

Así se vive la clasificación y la entrada en un programa como CHS. El acceso a las ayudas es difícil de comprender y de lograr. Se debe intentar logarlo de todas formas. Pero le rechazan el almuerzo de su hijo en la escuela, la inscripción de su hija en un programa de empleo joven, el subsidio a la vivienda y el crédito bancario, se debe responder la encuesta, se va a consultar los resultados, se obtiene un puntaje que certifica un estado de necesidad o que lo oculta, se debe resolver la alimentación fami-

11 "La Ficha de Protección Social es el único instrumento vigente que permite acceder a la red de beneficios sociales del Estado", en: http://www.fichaproteccionsocial.gob.cl . Visto el: 04/07/14. 
liar, llegar a fin de mes, intentar hacer que el Estado comprenda su realidad, resulta inimaginable pero existe la posibilidad de que el Estado la elija, hacerse invitar, tener suerte, ganar por fin: un lujo. Aquel es el relato vivencial de las mujeres beneficiarias: es una cuestión de suerte y de decepción, la incertidumbre articula la vinculación de los sujetos pobres con la política social del Estado. Al respecto, existe un conflicto lógico, insondable. El sistema de puntaje representa un artefacto ideológico que niega una realidad vivida y que limita el acceso a las ayudas sociales. No representa un indicador neutro, sino excluyente. Un conteo mal hecho, el producto de una burocracia torpe y lejana.

En el hecho fenomenológico, los puntajes no son verificables ni verosímiles. No producen certeza, contrariamente a lo que se pretende, porque carecen de relación con las experiencias de vida que suponen clasificar. El conflicto lógico tiene lugar entre la experiencia empírica de las mujeres demandantes de ayudas sociales y la técnica de conteo y clasificación de la vulnerabilidad: en un lado, la "realidad", en el otro, "la ficha”. En la experiencia cotidiana de las mujeres beneficiarias, no encontramos ni reconocimiento ni protección social: el Estado desconoce su realidad y, así, la atribución de ayuda no puede preverse. La clasificación social que despliegan los poderes públicos se transforma en un mecanismo de exclusión, una puerta difícil de atravesar, que ellas viven como incertidumbre, azar y suerte. Las ayudas sociales no significan un estatus de acceso al bienestar social, sino que una contingencia imprevisible.

Hay otros tópicos de la exclusión que se le articulan. Por ejemplo, la aceptación del rechazo:

Nos dieron miles de razones: que el puntaje lo daban desde allá, que era automático, que ellos no tienen nada que ver con el resultado final. Hay que aceptar lo que dicen, y punto (Tamara).

Hace años que estoy en lista de espera. Cuando voy a preguntar se enojan conmigo en la municipalidad, son irrespetuosos, la municipalidad ya no nos ayuda (Hilda).
Yo estaba tratando de conseguir ayuda para mi familia, para mis hijos: nos cerraron la puerta, "no hay ayuda aquí, no hay ayuda” nos dijeron. Después, ya no fui más (María).

No nos ayudan como debieran (Guadalupe).

En cierta forma, nos humillan cuando vamos, y no debería ser así (Pamela).

Del otro lado de la "realidad", el "allá": el Estado, que actúa como un otro poderoso, que cierra la puerta, que pone en lista de espera, que humilla, que pone un punto final. La relación con los trabajadores sociales y funcionario locales es reflejo de ese proceso. Allí toman forma relaciones de conflicto, de cercanía, de azar y de favor.

\section{Relaciones interpersonales y acce- so a las ayudas sociales}

A nivel municipal, la capacidad burocrática de influir en el proceso de atribución de las ayudas es baja por varios factores: los procesos de levantamiento de información social vienen estandarizados desde el nivel central, y allí se somete la información a procesos de filtrado y limpieza; la selección de los beneficiarios y las transferencias monetarias son gestionadas por el nivel central; las condiciones de profesionalización de los trabajadores sociales en el territorio y, específicamente, en el programa "Puente", para el caso chileno, son de muy bajo estándar en términos de formación, de condiciones salariales y contractuales, de permanencia en el empleo por lo mismo, y de recursos e infraestructura para el desempeño del trabajo de acompañamiento familiar. Sin embargo, los trabajadores sociales, en el territorio, pueden orientar otras ayudas sociales municipales (canastas básicas de alimentos, colchones y camas, "chapas" de madera y "mangas" plásticas para aislar paredes y techo, artefactos de cocina, recursos para la celebración de fiestas vecinales, etc.). Pueden, también, guiar a las usuarias respecto de cómo entregar información social en los procesos de encuesta y levantamiento, por 
ejemplo, sugiriéndoles declarar determinadas composiciones de núcleo familiar (sin hombre adulto, o como núcleo autónomo al interior de una familia extendida que comparte vivienda, ${ }^{12}$ etc.), a la vez que se vigila que no lo hagan. Los equipos territoriales y los profesionales a nivel individual gestionan diversificadamente su territorio dentro de un marco administrativo estructurado y de una estandarización de determinados programas, como "Puente", que preestablece la periodicidad de las visitas y sus protocolos, los contenidos y formatos de talleres grupales, el registro y la certificación de actividades mediante formularios, la intervención contingente en casos de emergencia, etc. Dentro de ese marco regulatorio, los trabajadores sociales hacen uso de un estrecho margen de discrecionalidad, con afán correctivo de ciertos aspectos del diseño de las políticas que implementan, motivados por favoritismos personales o pertenencia a redes barriales, como parte de las tramas políticas municipales en torno a la gestión del apoyo de las juntas de vecinos y a las elecciones locales.

En Chile, no encontramos un fenómeno generalizado de clientelismo local alimentado en base a la gestión municipal de las políticas de asistencia social - a diferencia de los casos emblemáticos de México (Hevia, 2010; 2007; Barba e Valencia-Lomelí, 2011) y de Argentiసे na (Vommaro e Quirós, 2011; Auyero, 2002) -, aunque no por ello se trata de una práctica ineสี่ xistente. Pero los márgenes de acción política municipal en torno a los programas de asistencia social son muy limitados por su dependencia directa, en algunos casos, del FOSIS, del MDS y de otros ministerios.

a La gestión local de las ayudas sociales y $\stackrel{\sim}{\circ}$ de CHS - "Puente" representa un proceso que, si bien exhibe ciertos márgenes discrecionales

${ }^{12}$ La "Ficha de Protección Social" se aplica por unidad familiar, y una variable determinante para su identificación es el hecho de cocinar separadamente de otros ocupantes

Te la misma vivienda. Según la composición de las fami-

fias, conviene a los demandantes de ayuda social declarar-

$\checkmark$ se como una sub-unidad familiar y demostrar que se coci-

na separadamente de los demás ocupantes de la vivienda,

exhibiendo que se tiene una cocinilla y un balón de gas,

por ejemplo. de gestión en el territorio, no es influyente en la selección de los beneficiarios ni opera a una magnitud política. Esto crea relaciones características entre usuarias de las ayudas sociales y trabajadores sociales en el terreno, así como con los funcionarios en el municipio, que no puede ser catalogado como clientelismo pero sí como favoritismo directo, a escala mínima de relación social, como se aprecia en las entrevistas. En ellas se combinan registros emocionales y normativos que dan cuenta de una ambigüedad relacional que obedece a un escenario en el que el rol de los trabajadores sociales en terreno y de los funcionarios municipales, a la vez que está regulado por marcos de estandarización de las intervenciones sociales, llega a constituirse como de facilitadores en el acceso a pequeños beneficios (canastas alimenticias, etc.). La relación se establece ambiguamente entre un plano personal, de empatía y de favoritismo con los trabajadores sociales, y una relación burocrática y oprimente con los funcionarios.

Son ásperos, son ásperos para recibirnos, nos esconden información, hacen como si estuvieran apurados todo el tiempo. (Hilda)

Me dijeron que no me la darían nunca [la cama para el hijo] y que ya estaba en mi ficha, que ya la habían pasado al computador y que no se borraría nunca porque ya estaba hecha. (Karen)

Tengo mala relación con las trabajadoras sociales. (Pamela)

Estoy muy agradecida con Gonzalo [funcionario municipal] y con la trabajadora social que me visitaba. Él es alguien muy preocupado por su gente. (Ruth)

En la municipalidad, la señorita me conoce bastante, yo sé que ella me va a ayudar. (Isabel)

Cualquier cosa que estén repartiendo, ella me llama, ella lo consigue para mí. (Katia)

Si una confía en la trabajadora social, esperas lo mejor de esa persona. (Guadalupe)

Depende de la voluntad de los funcionarios y de nosotras mismas, de nuestra manera de dirigirnos a ellos. (Ester)

¿Por qué hay personas que aceden a ayudas sociales y otras que no? Lo que interesa su- 
brayar aquí es la importancia dada a la relación de empatía con trabajadores sociales en terreno y funcionarios municipales al momento de explicarse, desde la perspectiva de las beneficiarias entrevistadas, las probabilidades de acceder a determinadas ayudas sociales. A pesar de la estandarización del trabajo de intervención social en el territorio, dispuesta desde el nivel central, las beneficiarias cifran su acceso a las ayudas sociales en función de la relación personal que consiguen - o no - establecer con los agentes de las ayudas sociales. Los propios trabajadores y trabajadoras sociales alimentan, en parte, esa relación cambiante con las beneficiarias, argumentando dificultad para regular la demanda que generan y para justificar ciertas decisiones de reparto, subrayando la escasez de recursos de que disponen para hacer intervención social directa. ${ }^{13}$ Así, los propios agentes de las políticas asistenciales en el territorio, ante su falta de prerrogativas en el proceso de selección de beneficiarios y de distribución de ayudas, alimentan los vínculos personales con las mujeres usuarias de la asistencia social como herramienta de legitimación necesaria para su trabajo de intervención (Rojas, 2014): si las usuarias están convencidas de que ellos no tienen capacidad decisional, entonces pierden credibilidad y legitimidad de acción. Al mismo tiempo, la propia intervención "psicosocial" que define el programa "Puente", y para la que genera material didáctico, burocrático y actividades específicas (talleres, jornadas, encuentros), cifra sus esperanzas en el éxito de las intervenciones en la capacidad de los trabajadores y trabadoras sociales de generar empatía y “contención” con las beneficiarias.

\section{REFLEXIONES FINALES}

Aquella es la experiencia cotidiana de ciudadanía social de las mujeres asistidas.

${ }^{13}$ Entrevistas y grupos de conversación con equipos de intervención directa o "acompañamiento" del programa "Puente" en comunas de Santiago, 2011 a 2013, ANR10-SUDS-0008 "Latinassist" .
Beneficiarse de ayudas sociales públicas es vivido como una improbabilidad, como el producto del azar, como un "lujo", como un premio que se "gana", como una "lotería", como "suerte", como una "invitación” exclusiva. Las beneficiarias buscan explicaciones para reducir su incertidumbre: se benefician de favores o del destino. El léxico de los derechos está prácticamente ausente en el discurso social de las mujeres beneficiarias de CHS y de otros programas de asistencia social. Los relatos expuestos reflejan lo que se denomina aquí como una ciudadanía asistencial determinada por un proceso hermético de calificación y por una vinculación tensionada con los agentes de las políticas en el territorio.

Si entendemos la ciudadanía social como pertenencia a la sociedad y acceso a la solidaridad pública, lo que se configura en torno de las ayudas sociales es un estatus de participación social inexistente, visto desde la relación social con el Estado, que ocurre en la interfaz que media entre la estructura de lo público y el sujeto ciudadano: una participación condicionada, subjetiva, azarosa, vista como favores. Aquella es la experiencia real que tiene lugar allí, en términos fenomenológicos y empíricos.

La inscripción en programas de ayuda social otorga un estatus incierto, azaroso, cambiante, insondable, fragmentado. La ciudadanía social de los pobres no es vivida como derechos adquiridos, sino que como una improbabilidad individual de acceder a las ayudas escasas y discrecionales. La ciudadanía asistencial es, en sí, excluyente y no refleja un acuerdo de integración, sino que tecnologías de gobierno de lo social o de la pobreza (Lautier, reeditado al portugués en esta publicación), en contextos de retracción de lo público y de expansión del mercado y del mercado de lo social (Georges \& Ceballos, en esta publicación). Para ello, se articulan mecanismos modulares, contingentes, selectivos y limitados de asignación de ayudas en contextos sociales de escasez material y de participación marginal en el mercado y en el trabajo (informalidad, 
endeudamiento, baja capacidad adquisitiva, carencia de ingresos autónomos y regulares). Estos dispositivos de gobierno intervienen desde el trabajo social en terreno, a través de agentes que cumplen un papel ambiguo entre el apadrinamiento, la condición funcionaria y la amistad - o la enemistad. Estos dispositivos también dirigen recursos monetarios a las economías familiares-femeninas. Las transferencias directas sostienen a un sujeto económico típicamente desaventajado por la desigualdad de género, pero a la vez reafirman su condición de género como madre responsable de la salud, educación y bienestar del núcleo familiar y, en particular, de los niños, ancianos y enfermos. Hay una individualización y una responsabilización explícitas, dramatizadas y justificadas por el relato político de la nueva asistencia social, de la mujer como sujeto funcional del bienestar social. Este referente de género no incorpora un relato emancipatorio de derechos de las mujeres, económicos primeramente, ni inseparables de éstos, todos los demás derechos (reproductivos, etc.). No se reconoce al individuo ciudadano mujer sino que al sujeto social madre. El "gobierno de los pobres" tiene lugar a través del género y en él se renuevan prácticas políticas usuales en América Latina, como el paternalismo (del Estado sobre los pobres, 는 sobre los niños y sobre las madres), el "emerخे gencialismo" y las acciones coyunturales, el control biopolítico de la asistencia social y de สี่ la medicina (Rojas, 2012; Illanes, 2006) y la clasificación de las poblaciones.

\section{REFERENCIAS}

AUYERO, Javier. Clientelismo político en Argentina: doble vida y negación colectiva. Perfiles Latinoamericanos, n. 20, p. 33-52, 2002.

BARBA, Carlos; VALENCIA-LOMELÍ, Enrique. Hipótesis no comprobadas y espejismos de las Transferencias Monetarias Condicionadas. In: BARBA, Carlos; COHEN, Néstor (Orgs.). Perspectivas críticas sobre la cohesión social. Desigualdad y tentativas fallidas de integración social en América Latina. Buenos Aires: CLACSO. 2011.

BARBA, Carlos. El nuevo paradigma de bienestar residual y deslocalizado. Reforma de los regímenes de bienestar en la OCDE, América Latina y México. Tesis doctoral en Sociología, ITESO, Guadalajara, México. 2003.

BARROS, Ricardo; HENRÍQUEZ, Ricardo; MENDOÇA, Rosane. A estabilidade inaceitável: desigualdade e pobreza no Brasil. Texto para discussão, n. 800. IPEA: Rio de Janeiro. 2001.

CEBALLOS, Marco; LAUTIER, Bruno. Les CCTP en Amérique latine: entre modélisation internationale et conjonctures politiques nationales. In: QUENAN, Carlos; VELUT, Sébastien; JOURCIN, Eric (Orgs.). Les enjeux du développement en Amérique latine: dynamiques socioéconomiques et politiques publiques. IdA/AFD: Paris. 2013.

CASTRO, Susana. Los dichos y los hechos. Las políticas sociales en el escenario neoliberal post-década del 90. El caso del Programa Jefas y Jefes de Hogar. Margen, n. 55. 2009. Disponible en http://www.margen.org/suscri/ margen55/castro.pdf. Visto en 03.03.2014

DONZELOT, Jacques. L'invention du social. Essai sur le déclin des passions politiques. Fayard: Paris. 1984.

DUHAU, Emilio. Las políticas sociales en América Latina: ¿del universalismo fragmentado a la dualización? Revista Mexicana de Sociología, México D. F., v. 59, n. 2, p. 185207. 1997.

FARIÑAS, María José. Ciudadanía "universal” versus ciudadanía "fragmentada". Cuadernos Electrónicos de Filosofía del Derecho, n. 2, p. 18-20. 1999.

FERNÁNDEZ, Oscar. Lo social y la política social. In: FERNÁNDEZ, Oscar (Org.). Política social y descentralización en Costa Rica. UNICEF/Universidad de Costa Rica: San José. 1998.

GARCÍA-CANCLINI, Néstor. Consumidores y ciudadanos. Conflictos multiculturales de la globalización. Grijalbo: México. 1995.

GARRETÓN, Manuel Antonio. Dimensiones políticas del estado social de derecho. In: ERAZO, Ximena; PAUTASSI, Laura; SANTOS, Antonia (Orgs.). Exigibilidad y realización de derechos sociales. Impacto en la política pública. LOM: Santiago. 2010

GOUGH, Ian. Welfare regimes in development contexts: global and regional analysis. In: GOUGH, Ian; WOOD, Geof (Orgs.). Insecurity and Welfare Regimes in Asia, Africa and Latina America. Social Policy in Development Contexts. Cambridge: Cambridge University Press. 2004.

HEVIA, Felipe. Usos politicos de programas sociales y nuevos intermediaries institucionales: el Programa Progresa/Oportunidades en el sur de Veracruz. Desacatos, México D. F. n. 34, p. 119-132. 2010.

HEVIA, Felipe. 2007. El programa Oportunidades y la construcción de ciudadanía. Tesis de Doctorado, CIESAS, Guadalajara, México. 2010.

IBÁÑEZ, Jesús. El regreso del sujeto: la investigación social de segundo orden. Siglo XXI: Madrid. 1994.

ILLANES, María Angélica. Cuerpo y sangre de la política. La construcción histórica de las Visitadoras Sociales (1887-1940). LOM: Santiago. 2006.

LAUTIER, Bruno. Gouvernement morale des pauvres et dépolitisation des politiques publiques en Amérique latine. Revue Tiers Monde, Paris, v. 2, n. 214, p. 169-186. 2013. DOI: $10.3917 / \mathrm{rtm} .214 .0169$.

Politiques de redistribution et de transferts sociaux. In: QUENAN, Carlos; VELUT, Sébastien (Orgs.). Les enjeux du développement en Amérique latine. Dynamiques socioéconomiques et politiques publiques. Ida/AFD: Paris. 2011. 
. La protection sociale dans le Brésil de Lula: trop de dépenses, ou trop peu de recettes? Revue Tiers Monde, Paris, v. 3, n. 175 , p. 527-554. 2003. DOI: 10.3917/ rtm.175.0527.

L'État-providence en Amérique latine: utopie légitimatrice ou moteur du développement? In: MARQUES-PEREIRA, Bérangère (Org.). L'Amérique Latine: vers la démocratie? Complexe: Bruxelles. 1993.

LOO-GUTIÉRREZ, Martín. La disciplina constitucional del principio de subsidiariedad en Italia y Chile. Revista de Derecho de la Pontificia Universidad Católica de Valparaíso, Valparaiso, n. 33, p. 391-426. 2009.

MARQUES-PEREIRA, Jaime. La crédibilité d'une politique anticyclique: les imperfections des marchés financiers à l'aune d'un déséquilibre externe structurel. In: LAUTIER, Bruno; MARQUES-PEREIRA, Jaime; SALAMA, Pierre. Régime de croissance, vulnérabilité financière et protection sociale en Amérique latine. Les conditions "marco" de l'efficacité de la lutte contre la pauvreté. CEPAL: Santiago. 2004.

MARSHALL, Thomas Humphrey. Ciudadanía y clase social. Revista Española de Investigaciones Sociológicas, Madrid, p. 297-344. 1997. URL: dialnet.unirioja.es/ descarga/articulo/760109.pdf

MELO-BORGES, Elcileni; SARMENTO-SEIXAS, Flávio. Redução da desigualdade: o impacto dos programas sociais de transferência de renda no país e em Goiás. Conjuntura Económica Goiana, n. 8, p. 11-18. 2006.

MORENO, Luis. Ciudadanía, desigualdad social y Estado de bienestar. Documento de Trabajo de la Unidad de Políticas Comparadas CSIC, n. 03-08. CSIC. 2003.

. Ciudadanos precarios. La "última red” de protección social. Barcelona: Ariel. 2000.

O’DONNELL, Guillermo. Acerca del estado, la democratización y algunos problemas conceptuales. Una perspectiva latinoamericana con referencia a países poscomunistas. In: Contrapuntos. Ensayos escogidos sobre autoritarismo y democratización. Paidós: Buenos Aires. 1997.

PAUGAM, Serge. Les formes contemporaines de la disqualification sociale. CERISCOPE Pauvreté. 2012. En http:/ceriscope.sciences-po.fr/pauvrete/content/part5/ lesformes-contemporaines-de-la-disqualification-sociale Visto el 12.04.2014.

ROJAS, Carolina. Applaudissez-vous, vous êtes des privilégiés! Une analyse politique et morale de l'assistance contemporaine au Chili. In: FASSIN, Didier; EIDELIMAN, Jean Sébastien (Orgs.). Économies morales contemporaines. La Découverte: Paris. 2012.

Sexuación y subjetivación en las prácticas de asistencia en Chile. Cadernos de Pesquisa, n. 152, v. 44, p. 312-333. 2014

SANTOS, Wanderley G. Ciudadania e Justiça. Rio de Janeiro: Campus. 1979.

SOARES, Sergei; OSÓRIO, Rafael; SOARES, Fabio; MEDEIROS; Marcelo; ZEPEDA, Eduardo. Conditional Cash Transfers in Brazil, Chile and Mexico: impacts upon inequality. Working paper, n. 35. International Policy Center for Inclusive Growth/UNPD: Rio de Janeiro. 2007.

SOJO, Carlos. La noción de ciudadanía en el debate latinoamericano. Revista de la CEPAL, Santiago, n. 76, 2002, p. 25-38. 2002.

SOJO, Ana. Vulnerabilidad social, aseguramiento y diversificación de riesgos en América Latina y el Caribe. Revista de la CEPAL, Santiago, n. 80, p. 121-140. 2003.

VOMMARO, Gabriel; QUIRÓS, Julieta. 'Usted vino por su propia decisión': repensar el clientelismo en clave etnográfica. Desacatos, México D.F, n.36, p.65-84. 2011.

YOUNG, Iris. Vida política y diferencia de grupo: una crítica del ideal de ciudadanía universal. In: CASTELLS, Carme (Org.). Perspectivas feministas en teoría política. Paidós: Barcelona. 1996. 


\section{CITIZENSHIP OR SOCIAL ASSISTANCE? the public policy project the experience of female beneficiaries of social assistance in Chile}

\section{Marco Ceballos}

From the Chilean experience, this article aims to examine the meaning of the notion of citizenship in the context of current assistance policies. At first time, we analyze their recent development as well as the logics and processes that explain the emergence and transformation of the known device "Chile Solidario" (CHS), emphasizing the constellation of social aid developed under his wing. In the second stage, the concept of citizenship is discussed from a sociological perspective, non-formal or normative, attempting to show, through the testimonies of women beneficiaries of social assistance policies, the empirical content of an assistance citizenship as it prevails in their life experiences.

KeYwords: Social policy. Social assistance. Latin America. Chile Solidario.

\section{LA CITOYENNETE OU L'AIDE SOCIALE? le projet de politique publique de l'expérience des femmes bénéficiaires de l'aide sociale au Chile}

\section{Marco Ceballos}

Autour de l'expérience chilienne, cet article interroge le sens pris par la notion de citoyenneté dans le cadre des politiques d'assistance sociale actuelles au Chili. Dans un premier moment, on analyse l'évolution récente de celles-ci ainsi que les logiques et les processus capables d'expliquer l'émergence et la transformation du assez connu dispositif "Chile Solidario” (CHS), mettant l'accent sur la constellation d'aides sociales qui se consolident à son insu. Dans un deuxième moment, le concept de citoyenneté est discuté depuis une perspective sociologique, non pas formelle ni normative, en essayant de montrer, à partir de témoignages de femmes bénéficiaires des politiques d'assistance sociales au Chili, le contenu empirique d'une citoyenneté assistantielle qui prévaut dans leurs expériences de vie.

Mots-clés: Politique sociale. L'aide sociale. L'Amérique Latine. Chile Solidario. Conditional Cash Transfer Programs (CCTP). Escola de Sociologia, Faculdade de Humanidades e Ciências Sociais, da Universidade Andrés Bello, UNAB, Viña del Mar, Valparaíso. Pesquisador no grupo de trabalho "Pobreza e políticas sociais" de CLACSO. Investigador-responsável do projeto FONDECYT/CONICYT n¹1140809 sobre "Mulheres trabalhadoras assistidas" (2014-2016). Investigador-responsável do capítulo chileno do projeto francolatinoamericano ANR-10-SUDS-0008 "Latinassist” (2010-2014) 